\title{
DETERMINATION OF THE COMPOSITION OF MULTILAYER PLASTIC PACKAGING WITH NIR SPECTROSCOPY
}

\author{
Xiaozheng Chen *, Nils Kroell, Alexander Feil and Thomas Pretz
}

Department of Processing and Recycling, RWTH Aachen University, Aachen, Germany

Article Info:
Received:
31 March 2020
Revised:
7 July 2020
Accepted:
28 July 2020
Available online:
5 October 2020
Keywords:
Near-infrared spectroscopy
Multilayer packaging
Polymer sorting
Recycling
Composition determination

\section{INTRODUCTION}

In the European Union, the largest proportion of produced plastics is used as packaging material, with about $40 \mathrm{wt} \%$ of the total amount. Among the packaging materials produced, $17 \mathrm{wt} \%$ are multilayer packaging films [Mumladze et al., 2018]. Flexible multilayer plastic packaging is becoming more and more popular especially for food and medical packaging due to the low energy consumption in production, low material requirements and the reduction in packaging weight [Butler, Morris; 2016]. Besides, multilayer packaging enables an extension of functional properties by adding additional layers, for example, a barrier layer against oxygen and water vapor [Butler, Morris; 2016].

Multilayer packaging consists of two or more layers of different materials, which depending on the production process, are either bonded with diffusion forces between layers or with special adhesives. There are different kinds of materials available, depending on the application area and functional requirements [Emblem, Hardwidge, 2012]. Each kind of material has its own physical and functional properties; Therefore, there is no general composition of multilayer packaging [Dixon, 2011]. The most commonly used flexible packaging film materials are low-density polyethylene (LDPE), polypropylene (PP) and polyethylene terephthalate (PET) [Wagner, 2016].

Plastic films separated from sorting plants often end in incineration or in low-quality products through downcycling [Plasticseurope, 2019]. To achieve a high recycling rate, even low quality waste streams, such as flexible multilayer packaging and mixed film waste, need to be recycled. Several companies have achieved post-industrial multilayer packaging recycling with different processing treatments. For example, the company APK GmbH from Germany has successfully recycled polyamide (PA) / polyethylene (PE) multilayer materials by using special solvents [APK $\mathrm{GmbH}, 2020]$. In case of post-consumer multilayer packaging waste, the recycling is not yet possible. One of the challenges is determining the composition and separating them from other packaging materials in post-consumer waste.

Near-Infrared (NIR) spectroscopy is the state-of-the-art technology in sorting plastic waste. It classifies materials according to their own characteristic spectrum [Siesler et al., 2002]. In this study, the composition determination of transparent post-consumer multilayer plastic packaging 
TABLE 1: Available monolayer materials and thickness.

\begin{tabular}{c|ccc} 
Material & Typical thickness $(\boldsymbol{\mu m})$ [Dixon, 2011] & Colour & Analysed thickness $(\boldsymbol{\mu m})$ \\
\hline OPP & $15-50$ & transparent & $15,20,35^{\star}$ \\
\hline LDP & $25-100$ & transparent & $30,50,70,100 *$ \\
\hline PET & $12-50$ & transparent & $12^{\star}, 23,45^{\star}, 50^{\star}$ \\
\hline
\end{tabular}

*printed sample available; OPP: oriented PP

waste is investigated with NIR spectroscopy by comparing the spectra of monolayer with two and three-layers samples. Based on this, the composition of two-layer samples was analysed and determined. In addition, influences of layer thickness, material proportions, layer sequence and printed surfaces were investigated.

\section{MATERIAL AND METHODS}

In Germany, 2-dimensional (2D) plastic, e.g. films, and 3-dimensional plastics are collected in lightweight packaging waste. In most sorting plants, films are firstly separated from the input waste stream with air classifiers or ballistic separators for further processing or transportation [Pretz et al., 2020]. Therefore, multilayer packaging needs to be separated from other 2D plastics.

\subsection{Material selection}

As adhesives are not necessary in multilayer production and the thickness of adhesives layer is typically much lower than the plastic layers [Emblem, Hardwidge, 2012], samples without adhesives are used. The investigated material types are LDPE, PP and PET, since they are most often used in flexible packaging films [Wagner, 2016]. Typical thickness in packaging film plastics as well as available monolayer material, colours and thicknesses are shown in Table 1. Multilayer samples were two or more layers of monolayer samples. The samples are virgin material and were produced by the company Estiko-Plastar AS (Tartu, Estonia).

\subsection{Spectral analysis}

The detection of multilayer plastics and the sorting ability were determined through spectral analysis with NIR spectroscopy. The NIR sensor in this study is a Helios-G2-320 from EVK DI Kerschhaggl GmbH (Raaba, Austria) with a spectral range of 900 to $1700 \mathrm{~nm}$ and a spatial resolution of $0.8 \mathrm{~mm} /$ pixel. Halogen lamps are used as emitters. The reflection of radiation from the surface is captured by the NIR sensor, see Figure 1. The difference in NIR spectra is represented by changing of the intensities of the reflected radiation. Consequently, the first derivative of the reflected radiation was used for analysis and the samples were then pixel-based classified with support vector machine algorithms based on the characteristic peaks (position and level).

Usually in sorting plants, the background of a NIR-based sorter is a black conveyor belt. Black materials reflect less radiation and can thus be easily distinguished from nonblack objects. However, since most multilayer packaging materials are transparent and have low thickness, the intensity of the reflected radiation is relatively low and the detection is limited. To demonstrate a general feasibility of the composition determination for flexible multilayer plastic packaging, the used background was a homogenous paper in dark green, which is NIR active and has its own characteristic spectrum. The detection and classification of materials are therefore based on the differences between the spectra of samples and of the used NIR-active paper.

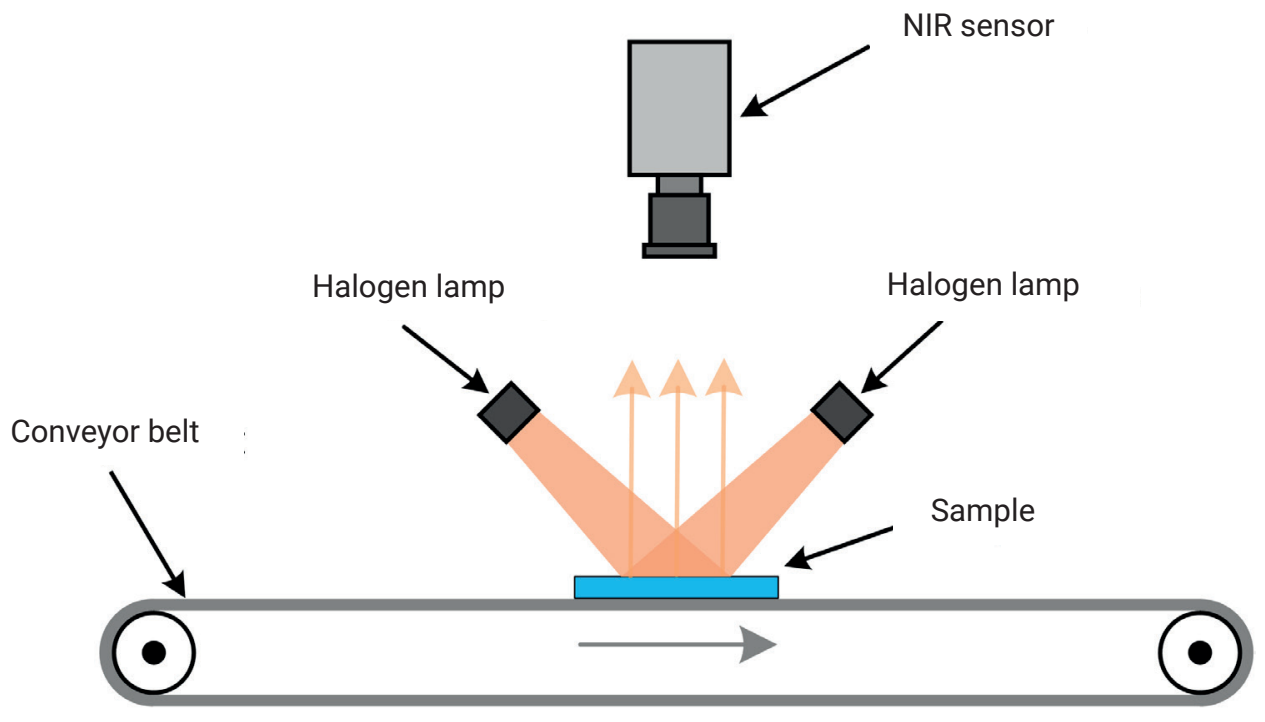

FIGURE 1: Schematic sketch of NIR sensor and halogen lamps arrangement. 


\section{RESULT AND DISCUSSION}

In order to determine the detectability of different multilayer samples, spectra of background material and monolayers were captured. The spectra of multilayer samples were then compared with the ones of the background and corresponding monolayers to determine whether the difference is great enough to be detected and classified. Figure 2 shows mean spectra of background, transparent OPP with a thickness of $35 \mu \mathrm{m}$, transparent LDPE with $30 \mu \mathrm{m}$ and two layers combinations as an example.

As Figure 2 shows, all layers could be detected, since their spectra differ from the spectrum of the background. The two-layer samples could be distinguished from the monolayer samples by the position of peaks, e.g. in the wavelength area 1200-1300 nm and about 1460-1550 nm. However, the spectra shown in Figure 2 are only the mean values of some selected pixels and classification process of all pixels is necessary for determining the sorting abil- ity. The results in Figure 3 show that the samples were successfully classified into their right classes with an accuracy of more than $95 \%$. The spectra of other mono- and two-layer materials were also compared with each other and the classification results are similar. Diverse compositions show spectra with slight differences and it was possible to classify more than $87 \%$ of all pixels to the right classes.

Additionally, three-layers samples exhibited a difference to mono- and two-layers ones in spectra (figure 2) and the classification according to the composition was also possible. The spectrum of three-layers PET/OPP/LDPE shows a difference at the wavelength from 1160 to 1300 $\mathrm{nm}$ and at about $1400 \mathrm{~nm}$ to the PET monolayer. At about $1680 \mathrm{~nm}$, the negative peak differs this three-layers sample from OPP and LDPE. With these characteristics, the samples were possible to be classified to their classes with an accuracy of $89 \%$.

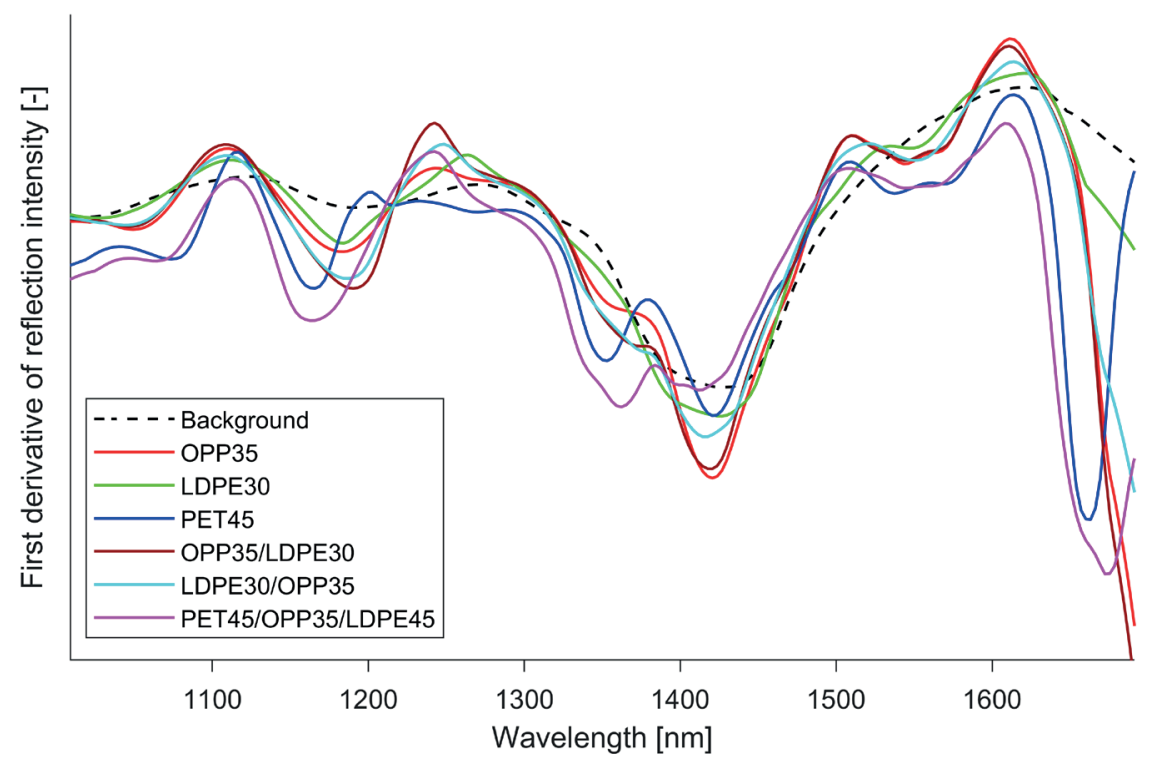

FIGURE 2: Mean spectra of background, OPP35, LDPE30, PET45, OPP35/LDPE30, LDPE30/OPP35 and PET45/OPP35/LDPE30. (Sample name is material with thickness, e.g. OPP35/LDPE30 is two-layer sample with one OPP layer with a thickness of $35 \mu \mathrm{m}$ and one LDPE Layer with a thickness of $30 \mu \mathrm{m}$, the OPP layer is exposed to NIR sensor).

OPP35

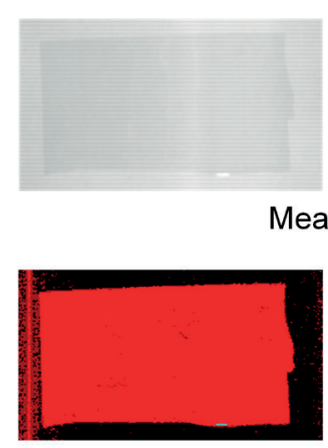

LDPE30
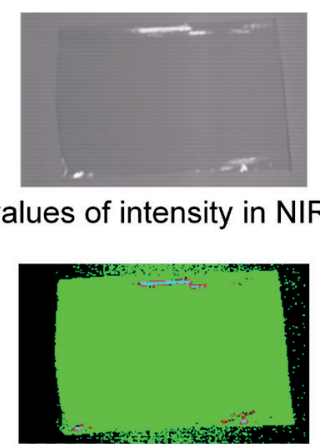

Classification result

\section{LDPE30/OPP35}
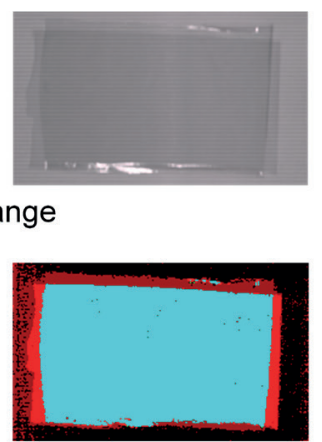

Pixels classified as LDPE

Pixels classified as OPP+LDPE

FIGURE 3: Classification results of OPP35, LDPE30 and OPP35/LDPE30. 


\subsection{Effects of individual layer thickness}

To determine the influence of thickness and proportion of each material on classification, samples with one layer of OPP with a thickness of $35 \mu \mathrm{m}$ and one layer of LDPE with 30, 50, 70 and $100 \mu \mathrm{m}$ thickness each were analysed. Figure 4 shows that with increasing thickness of the LDPE layer, peaks and their position of the multilayer spectrum are getting more similar to the spectrum of LDPE monolayer. Analysis of other multilayer samples (e.g. PET with different thickness of LDPE) showed similar trends. Besides, the thicker the material, the greater the intensity of the peaks are. However, in this case, the proportion of LDPE increases with the overall thickness of the analysed sample, such that the influence of proportion on intensity could not be demonstrated. The classification results indicated that all the two-monolayer samples were able to be classified with an accuracy of $96 \%$.

\subsection{Effects of printing colour and layer sequence}

For transparent samples, the sequence of layers did not influence the spectra, see Figure 2. The mean spectra of OPP35/LDPE30 in dark red and LDPE30/OPP35 in cyan in Figure 2 show a slight difference, but it was not sufficient to classify them into two classes, as almost half of the pixels were classified to wrong classes. Besides, there is no difference in the spectra and classification results regarding which side of the layer is exposed to the NIR sensor. For layers which are not glossy, the spectra of the samples with one layer printed are very similar to the transparent ones. This means that the detection and classification are independent on whether the samples are printed or not. Contrarily, in case of glossy samples, they could only be detected on the non-printed side, as the radiation is fully reflected from the glossy side and thus, the spectra could not be classified.

\section{CONCLUSIONS}

Through the analysis of monolayer, two- and three-layers samples with NIR spectroscopy, it has been demonstrated that different multilayer samples can be distinguished from each other by the classification of the captured spectra with support vector machine algorithms under laboratory conditions. Samples with different composition (OPPI LDPE, OPP\PET, LDPE\PET and OPP\PET\LDPE) have been analyzed and the captured spectra showed a difference to other multilayer and to the monolayer materials. The sequence of layers does not influence the spectra of samples, but there is a difference in spectra when glossy samples are printed. With increasing layer thickness, the intensity of peaks in the captured spectra is higher, which makes it easier to classify them. With the increase of proportion of one polymer, spectra are getting more similar to the spectrum of this material. Consequently, sorting materials according to their main composition might be possible for post-consumer multilayer plastic packaging.

However, all the tests in this study are in laboratory conditions and the sample materials were virgin production material and did not come from post-consumer waste streams. In practical sorting processes, post-consumer packaging plastics often have impurities on the surface, which, on the one hand, brings benefit as the samples are less glossy and they could be detected. On the other hand, NIR-active impurities can influence the spectra of the materials and make the classification more difficult. In further research, the impact of impurities and the detection and classification of post-consumer multilayer plastic packaging should be analyzed. In addition, further research is required to evaluate whether these results can be transferred to the NIR sorting of post-consumer waste streams.

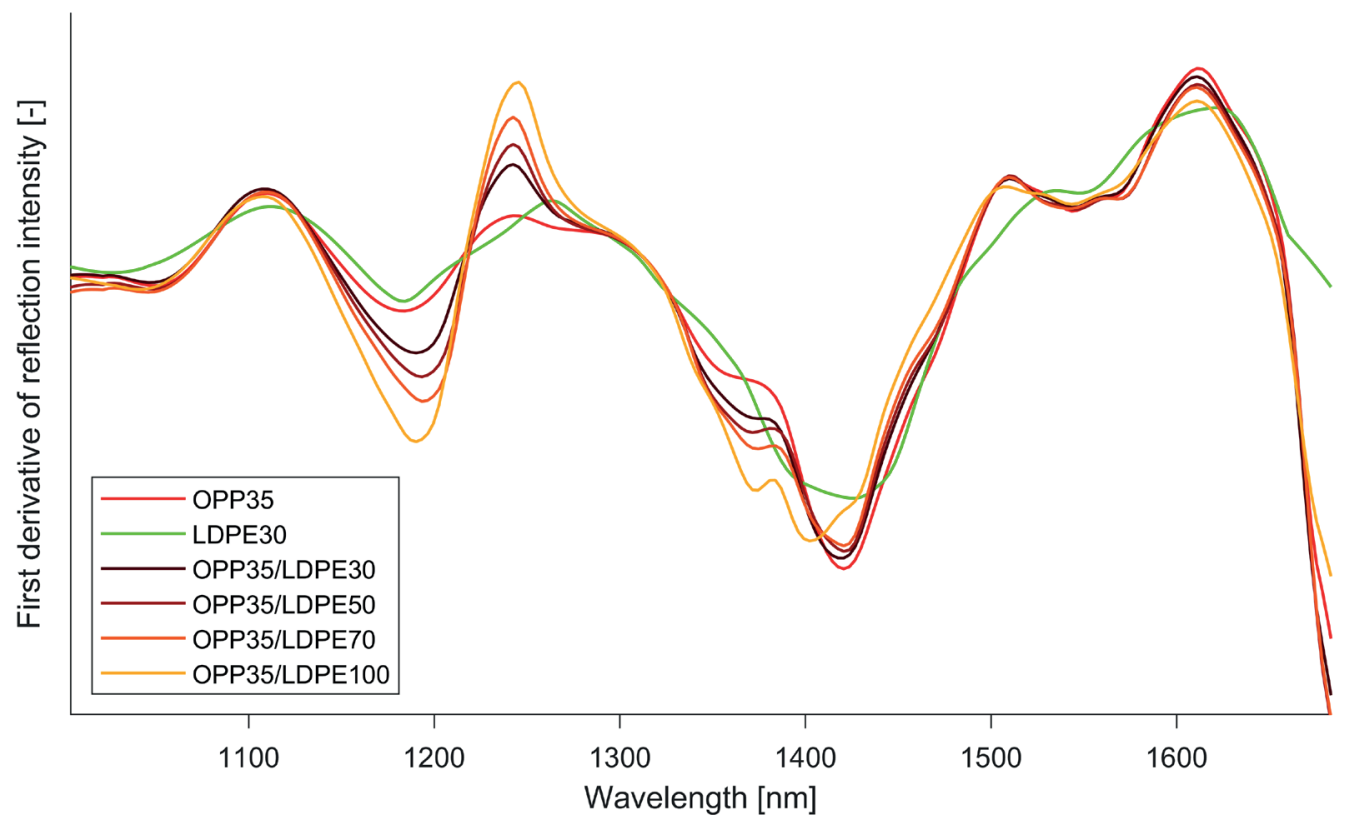

FIGURE 4: Mean spectra of samples with OPP $35 \mu \mathrm{m}$ and LDPE 30, 50, 70 and $100 \mu \mathrm{m}$. 


\section{REFERENCES}

APK GmbH: Newcycling. https://www.apk-ag.de/newcycling/. Retrieved January 26, 2020

Butler, T. I., Morris, B. A., (2016): PE-Based Multilayer Film Structures Wagner, J. R. Jr.: Multilayer Flexible Packaging; Oxford (Elsevier).

Dixon, J. (2011): Packaging materials: 9. Multilayer packaging for food and beverages; Brussels.

Emblem, A., Hardwidge, M. (2012): Adhesives for packaging - Emblem, A., Emblem, H. (Ed.): Packaging Technology; Sawston. DOI: 10.1533/9780857095701.2.381.
Mumladze, T., Yousef, S., Tatsrisnts, M., Kriūkienė, R., Makarevivius, V., Lukošiūtè, S.-I., Bendikiene, R., Denafas, G. (2018): Sustainable approach to recycling of multilayer flexible packaging using switchable hydrophilicity solvents - Green Chem., 2018, 20, S. 3604-3618; Aachen.

PLASTICSEUROPE (2019): Plastics - the Facts 2019.

Pretz, T., Raulf, K., Quicker, P. (2020): Waste, 4. Recycling - Ullmann's Encyclopedia of Industrial Chemistry (pp. 1-39); Weinheim (WILEY-VCH)

Siesler, H. W., Ozaki, Y., Kawata, S., Heise, H. M. (2002): Near-Infrared Spectroscopy; Weinheim (WILEY-VCH)

Wagner, J. R. Jr. (2016): Multilayer Flexible Packaging (2nd Edition); Oxford (Elsevier). 